\title{
PROBABILITIES OF VERY LARGE DEVIATIONS
}

\author{
STEPHEN A. BOOK \\ (Received 21 December 1976; revised 13 April 1977) \\ Communicated by $\mathbf{J}$. Gani
}

\begin{abstract}
If $\left\{X_{n}: 1 \leqq n<\infty\right\}$ are independent, identically distributed random variables having $E\left(X_{1}\right)=$ 0 and $\operatorname{Var}\left(X_{1}\right)=1$, the most elementary form of the central limit theorem implies that $P\left(n^{-\frac{1}{2}} S_{n} \geqq z_{n}\right) \rightarrow 0$ as $n \rightarrow \infty$, where $S_{n}=\sum_{k=1}^{n} X_{k}$, for all sequences $\left\{z_{n}: 1 \leqq n<\infty\right\}$ for which $z_{n} \rightarrow x$. The probability $P\left(n^{-\frac{1}{2}} S_{n} \geqq z_{n}\right)$ is called a "large deviation probability", and the rate at which it converges to 0 has been the subject of much study. The objective of the present article is to complement earlier results by describing its asymptotic behavior when $n^{-\frac{1}{2}} z_{n} \rightarrow \infty$ as $n \rightarrow \infty$, in the case of absolutely continuous random variables having moment-generating functions.
\end{abstract}

Subject classification (Amer. Math. Soc. (MOS) 1970): primary 60F10, secondary 60F15.

Keywords: large deviations, asymptotic expansions, moment-generating functions, laws of large numbers.

\section{Introduction}

If $\left\{X_{n}: 1 \leqq n<\infty\right\}$ is a sequence of independent, identically distributed (iid) random variables having the standard normal distribution, and $\left\{z_{n}: 1 \leqq\right.$ $n<\infty\}$ is a sequence of positive numbers such that $z_{n} \rightarrow \infty$ as $n \rightarrow \infty$, an elementary large deviation result asserts that

$$
P\left(n^{-\frac{1}{2}} S_{n} \geqq z_{n}\right)=(2 \pi)^{-\frac{1}{2}} z_{n}^{-1} \exp \left\{-\frac{1}{2} z_{n}^{2}\right\}\left\{1+O\left(z_{n}^{-2}\right)\right\}
$$

where $S_{n}=\sum_{k=1}^{n} X_{k}$. A proof can be found on p. 175 of Feller (1968).

Theorems extending the above assertion to non-normal random variables divide basically into two classes: (1) where the tail of the common distribution function is asymptotic to $x^{-\alpha}$ for some $\alpha>0$ as $x \rightarrow \infty$; and (2) where the underlying random variables have a moment-generating function (mgf) $\phi(t)$ existing in a nondegenerate interval. Major results belonging to class (1) are those appearing in Heyde (1968), Rohatgi (1973), and on pp. 255-258 of Ibragimov and Linnik (1971). As a rule, these results show that 
$P\left(\beta_{n}^{-1} S_{n} \geqq z_{n}\right)=n P\left(X_{1} \geqq \beta_{n} z_{n}\right)\{1+o(1)\}$ as $n \rightarrow \infty$, where the $\beta_{n}$ 's are normalizing factors. The main theorem of the present article falls into class (2).

Throughout the remainder of the paper, we deal only with random variables normalized so that $E\left(X_{1}\right)=0$ and $\operatorname{Var}\left(X_{1}\right)=1$.

The first general result of class (2) was obtained by Cramér (1938), who worked with $z_{n}$ 's such that $n^{-\frac{1}{2}} z_{n} \rightarrow 0$ as $n \rightarrow \infty$, as improved by Petrov (1954). In the same article, Cramér established a large deviation result for "strongly non-lattice" random variables when $z_{n}=c n^{\frac{1}{2}}$ for some constant $c>0$. This result was later extended by Bahadur and Ranga Rao (1960) to random variables of all types.

The objective of the present article is to derive an asymptotic representation of the probability $P\left(n^{-\frac{1}{2}} S_{n} \geqq z_{n}\right)$ when the $z_{n}$ 's tend to $\infty$ faster than $n^{\frac{1}{2}}$. The proof, based on methods due to Bahadur and Ranga Rao (1960), requires that certain moments of an "associated" distribution must be uniformly bounded. Furthermore, the faster $z_{n}$ goes to $\infty$, the higher is the order of those moments that must be bounded. To insure this degree of increasingly normal-like behavior, we require that the underlying mgf exist on the whole real line, and we extend a theorem of Baum, Katz, and Read (1962) to show that this requirement is necessary.

\section{Preliminaries}

We consider a sequence $\left\{X_{n}: 1 \leqq n<\infty\right\}$ of iid absolutely continuous random variables having $E\left(X_{1}\right)=0, \quad \operatorname{Var}\left(X_{1}\right)=1$, and $\operatorname{mgf} \phi(t)=$ $E\left(\exp t X_{1}\right)<\infty$ for all positive values of $t$. For a sequence $\left\{z_{n}: 1 \leqq n<\infty\right\}$ of real numbers such that $n^{-\frac{1}{2}} z_{n} \rightarrow \infty$ as $n \rightarrow \infty$, we have for $S_{n}=\sum_{k=1}^{n} X_{k}$ that

$$
P\left(n^{-\frac{1}{2}} S_{n} \geqq z_{n}\right)=P\left(\sum_{k=1}^{n} Y_{n k} \geqq 0\right)
$$

where $Y_{n k}=n^{-\frac{1}{2}} X_{k}-n^{-1} z_{n}$. The distribution function (df) of $Y_{n k}$ is given by

$$
G_{n k}(y)=P\left(Y_{n k} \leqq y\right)=F\left(n^{\frac{1}{2}} y+n^{-\frac{1}{2}} z_{n}\right),
$$

where $F(x)=P\left(X_{1} \leqq x\right)$ is the df of $X_{1}$. Denoting the density function of $X_{1}$ by $f(x)=F^{\prime}(x)$, we see that the density function of $Y_{n k}$ is

$$
g_{n k}(y)=G_{n k}^{\prime}(y)=n^{\frac{1}{2}} f\left(n^{\frac{1}{2}} y+n^{-\frac{1}{2}} z_{n}\right) .
$$

The mgf of $Y_{n k}$ can be expressed as

$$
\psi_{n k}(t)=E\left(\exp t Y_{n k}\right)=e^{-n^{-1} z_{n} t} \phi\left(n^{-\frac{1}{2}} t\right) .
$$

We define for each $h, 0 \leqq h<\infty$, an "associated" random variable $\bar{Y}_{n k}(h)$ by 
its $\mathrm{df}$

$$
d \bar{G}_{n k}(y)=\left\{e^{h y} / \psi_{n k}(h)\right\} d G_{n k}(y) .
$$

The mgf of $\bar{Y}_{n k}(h)$ is

$$
\bar{\psi}_{n k}(t)=\frac{\psi_{n k}(t+h)}{\psi_{n k}(h)}=\left\{\frac{\phi\left(n^{-\frac{1}{2}} t+n^{-\frac{1}{2}} h\right)}{\phi\left(n^{-\frac{1}{2}} h\right)}\right\} e^{-n^{-1} z_{n} t} .
$$

We further set $\bar{S}_{n}=\sum_{k=1}^{n} \bar{Y}_{n k}$ and then

$$
\bar{G}_{n}(y)=P\left(\bar{S}_{n} \leqq y\right) .
$$

A change of variable and an integration by parts yield the following lemma:

(1.2) Lemma. For every $h \geqq 0$,

$$
P\left(n^{-\frac{1}{2}} S_{n} \geqq z_{n}\right)=e^{-z_{n} h} \phi^{n}\left(n^{-\frac{1}{2}} h\right) h \int_{0}^{\infty} e^{-h y}\left\{\bar{G}_{n}(y)-\bar{G}_{n}(0)\right\} d y .
$$

Using the fact that $\bar{S}_{n}(h)$ has $\mathrm{mgf}$

$$
\bar{\psi}_{n}(t)=\prod_{k=1}^{n} \bar{\psi}_{n k}(t)=\left\{\frac{\phi\left(n^{-\frac{1}{2}} t+n^{-\frac{1}{2}} h\right)}{\phi\left(n^{-\frac{1}{2}} h\right)}\right\}^{n} e^{-z_{n} t}
$$

and defining

$$
Q(t)=\phi^{\prime}(t) / \phi(t)
$$

we obtain the next lemma:

(1.4) Lemma. $E\left(\bar{S}_{n}(h)\right)=n^{\frac{1}{2}} Q\left(n^{-\frac{1}{2}} h\right)-z_{n}$

and

$$
\operatorname{Var}\left(\bar{S}_{n}(h)\right)=Q^{\prime}\left(n^{-\frac{1}{2}} h\right) .
$$

Using the next lemma, we choose a particular sequence $\left\{h_{n}: 1 \leqq n<\infty\right\}$ of $h$ 's. For notational ease, we write $A \leqq \overline{\lim }_{n \rightarrow \infty} C_{n} \leqq B$ to indicate that $A \leqq \liminf _{n \rightarrow \infty} C_{n} \leqq \lim \sup _{n \rightarrow \infty} C_{n} \leqq B$.

(1.5) Lemma. If

$$
\lim _{t \rightarrow \infty} Q(t)=\infty
$$

and

$$
\text { there exist numbers } \sigma_{1}^{2} \text { and } \sigma_{2}^{2} \text { such that }
$$

$$
0<\sigma_{1}^{2} \leqq Q^{\prime}(t) \leqq \sigma_{2}^{2}<\infty
$$

for all sufficiently large $t$, then, for every sufficiently large $n$, there exists a 
solution $h=h_{n}$ to the equation $E\left(\bar{S}_{n}(h)\right)=0$,

$$
0<\sigma_{1}^{2} \leqq \varlimsup_{n \rightarrow x} h_{n}^{-1} z_{n} \leqq \sigma_{2}^{2}<\infty
$$

and

$$
0<\sigma_{1}^{2} \leqq \varlimsup_{n \rightarrow \infty} \bar{\sigma}_{n}^{2} \leqq \sigma_{2}^{2}<\infty
$$

where $\bar{\sigma}_{n}^{2}=Q^{\prime}\left(n^{-\frac{1}{2}} h_{n}\right)$.

Proof. In view of Lemma 1.4, $E\left(\bar{S}_{n}(h)\right)=0$ if and only if $Q\left(n^{-\frac{1}{2}} h\right)=$ $n^{-\frac{1}{2}} z_{n}$. By condition (1.5a) and the continuity of $Q$, there must exist for each $n$ an $h_{n}$ with $Q\left(n^{-\frac{1}{2}} h_{n}\right)=n^{-\frac{1}{2}} z_{n}$. Therefore

$$
h_{n}=n^{\frac{1}{2}} Q^{-1}\left(n^{-\frac{1}{2}} Z_{n}\right)
$$

as $Q$ is one-to-one since $Q^{\prime}(t)>0$ for all $t$, being the variance of a nondegenerate random variable. Condition (1.5a) now implies that $h_{n} \rightarrow \infty$ as $n \rightarrow \infty$ because $n^{-\frac{1}{2}} z_{n} \rightarrow \infty$. Furthermore

$$
z_{n}^{-1} h_{n}=\left(n^{-\frac{1}{2}} z_{n}\right)^{-1} Q^{-1}\left(n^{-\frac{1}{2}} z_{n}\right),
$$

so that, by L'Hôpital's Rule for subsequences $\left\{n_{k}: 1 \leqq k<\infty\right\}$,

$$
\lim _{k \rightarrow \infty} z_{n_{k}}^{-1} h_{n_{k}}=\lim _{k \rightarrow \infty} u_{k}^{-1} Q^{-1}\left(u_{k}\right)=\lim _{k \rightarrow \infty}\left\{Q^{\prime}\left(Q^{-1}\left(u_{k}\right)\right)\right\}^{-1}
$$

where $u_{k}=n_{k}^{-\frac{1}{2}} z_{n_{k}}$. Assertion (1.5c) follows. From (1.5c) and the fact that $n^{-\frac{1}{2}} z_{n} \rightarrow \infty$, assertion (1.5d) follows.

The next step in the analysis requires an asymptotic expansion of the distribution function $\bar{G}_{n}(y)=P\left(\bar{S}_{n} \leqq y\right)$ that appears in the statement of Lemma 1.2. The Cramér asymptotic expansion below is discussed in more detail on pp. 1224-1227 of Book (1972) in a slightly more general form. A complete proof is available in Chap. 4 of Book (1970).

(1.7) Cramér's Asymptotic Expansion. If $\left\{X_{n k}: 1 \leqq k<\infty, 1 \leqq n<\infty\right\}$ is a triangular array of random variables such that

(1.7a) $X_{n 1}, \cdots, X_{n n}$ are independent for each $n$;

(1.7b) $E\left(X_{n k}\right)=0$ for all $k$ and $n$;

(1.7c) $\sum_{k=1}^{n} \operatorname{Var}\left(X_{n k}\right)=1$ for all $n$;

(1.7d) $E\left(\left|X_{n k}\right|^{m}\right)=\beta_{m n k}<\infty$ for all $k$ and $n$ and some $m \geqq 3$;

(1.7e) each $X_{n k}$ has absolutely continuous distribution $F_{n k}(x)$;

(1.7f) each density $f_{n k}(x)=F_{n k}^{\prime}(x)$ has finite total variation $v_{n k}$; and

(1.7g) if $\quad \Omega_{n}=\left\{k: 1 \leqq k \leqq n, \quad v_{n k} \leqq(3 n)^{\frac{1}{2}} /\left(32 \rho_{m n}^{3 / m}\right)\right\}$, where $\rho_{m n}=$ 
$n^{(m / 2)-1} \sum_{k=1}^{n} \beta_{m n k}$, then every sequence $\left\{n_{r}: 1 \leqq r \leqq \infty\right\}$ of positive inte gers contains a subsequence $\left\{n_{p}: 1 \leqq p<\infty\right\}$ such that either

(A) $\lim _{p \rightarrow \infty}\left(\log n_{p}\right)^{-1} \operatorname{card}\left(\Omega_{n_{p}}\right)=\infty$ or

(B) $\lim _{p \rightarrow \infty}\left(n_{p}^{-1} \rho_{m n_{p}}^{6 / m} \log n_{p}\right)^{-1} \Sigma_{k \in \Omega_{n p}^{c}} v_{n_{p} k}^{-2}=\infty$,

then, if $F_{n}(x)=P\left(\sum_{k=1}^{n} X_{n k} \leqq x\right)$,

$$
F_{n}(x)=\Phi(x)+\sum_{j=1}^{m-3} n^{-j / 2} p_{3 j-1, n}(x) e^{-x^{2 / 2}}+R_{m n}(x),
$$

where $\Phi(x)=(2 \pi)^{-\frac{1}{2}} \int_{-\infty}^{x} e^{-u^{2} / 2} d u,\left|R_{m n}(x)\right|<M^{*} n^{1-(m / 2)} \rho_{m n}^{(6 / m)-3}$ for $M^{*}$ independent of $n$ and $x$, and $p_{3 j-1, n}(x)$ is a polynomial of degree $3 j-1$ in $x$, further explicit details about which can be found in Book (1970) and in Corollary (1.13) below.

In order to apply Cramér's asymptotic expansion (1.7) to our situation, we define

$$
X_{n k}=\bar{\sigma}_{n}^{-1} \bar{Y}_{n k}\left(h_{n}\right) \text {, }
$$

where $E\left(\bar{Y}_{n k}\left(h_{n}\right)\right)=0$ and $\bar{\sigma}_{n}^{2}=\sum_{k=1}^{n} \operatorname{Var}\left(\bar{Y}_{n k}\left(h_{n}\right)\right)$ because the $\bar{Y}_{n k}\left(h_{n}\right)$ 's, $1 \leqq k \leqq n$, are iid for each $n$. It follows immediately that conditions (1.7a), $(1.7 \mathrm{~b})$, and (1.7c) hold. Our objective in the next several lemmas will be to specify situations in which the remaining conditions of (1.7) hold. We first recall from calculus the following fact, which can be proved using mathematical induction:

(1.8) Lemma. If $f(x)$ and $g(x)$ are $n$-times differentiable and $y=f(x) g(x)$, then $d^{n} y / d x^{n}=\sum_{k=0}^{n}\left(\begin{array}{l}n \\ k\end{array}\right) f^{(k)}(x) g^{(n-k)}(x)$.

Applying Lemma 1.8 to the mgf $\bar{\psi}_{n k}(t)$ of $\bar{Y}_{n k}=\bar{Y}_{n k}\left(h_{n}\right)$, taking note of (1.1), (1.3), and (1.6), and setting $t=0$, we obtain

(1.9) LEMMA. $E\left(\bar{Y}_{n k}^{m}\right)=n^{-m / 2} G^{(m)}\left(n^{-\frac{1}{2}} h_{n}\right)$,

where

$$
G^{(m)}(t)=\sum_{j=0}^{m}\left(\begin{array}{c}
m \\
j
\end{array}\right)(-1)^{j}\left(\frac{\phi^{\prime}(t)}{\phi(t)}\right)^{\prime} \frac{\phi^{(m-j)}(t)}{\phi(t)} .
$$

The next three lemmas follow immediately.

(1.10) LEMma. If $m \geqq 4$ is an even positive integer, then

$$
\beta_{m n k}=E\left(X_{n k}^{m}\right)=n^{-m / 2} \bar{\sigma}_{n}^{-m} G^{(m)}\left(n^{-\frac{1}{2}} h_{n}\right) .
$$

(1.11) LEMMA. $\rho_{m n}=n^{(m / 2)-1} \sum_{k=1}^{n} \beta_{m n k}=\bar{\sigma}_{n}^{-m} G^{(m)}\left(n^{-\frac{1}{2}} h_{n}\right)$.

(1.12) Lemma. If conditions (1.5a) and (1.5b) hold, together with

(1.12a) for an even positive integer $m \geqq 4$, there exist numbers $\gamma_{1}$ and $\gamma_{2}$ (depending on $m$ ) such that 


$$
0<\gamma_{1} \leqq G^{(m)}(t) \leqq \gamma_{2}<\infty
$$

for all sufficiently large $t$,

then

$$
0<\sigma_{2}^{-m} \gamma_{1} \leqq \varlimsup_{n \rightarrow x} \rho_{m n} \leqq \sigma_{1}^{-m} \gamma_{2}<\infty
$$

The following corollary presents the Cramér asymptotic expansion in the form we will use.

(1.13) Corollary. If $\left\{X_{n k}: 1 \leqq k \leqq n, 1 \leqq n<\infty\right\}$ is a triangular array of random variables satisfying the conditions of Cramér's Asymptotic Expansion (1.7), as well as (1.12b), then expansion (1.7h) holds with

$$
\left|R_{m n}(x)\right|<M n^{-(m-2) / 2},
$$

where $M$ is independent of $n$ and $x$,

$$
p_{3 j-1, \mathrm{n}}(x)=e^{x^{2 / 2}} P_{j n}(-\Phi)
$$

is a polynomial of degree $3 j-1$ in $x$, where

$$
P_{i n}(-\Phi)=\sum_{q=1}^{j}(-1)^{j+2 q} c_{q j n} \Phi^{(j+2 q)}(x),
$$

where the $c_{y j n}$ 's are uniformly bounded for all sufficiently large $n$, the bound depending only on $m, \sigma_{1}$, and $\gamma_{2}$, and

$$
P_{i n}(i t)=\sum_{q=1}^{i} c_{q j n}(i t)^{j+2 q}=e^{\ell^{2 / 2}} \int_{-\infty}^{\infty} e^{i t x} d P_{i n}(-\Phi) .
$$

(The proofs of these facts can be found in Chapter 4 of Book (1970)).

Finally the following lemma isolates the most crucial step of the proof of the main theorem:

(1.14) LeMmA. If $m \geqq 4$ is an even integer and $\left\{z_{n}: 1 \leqq n<\infty\right\}$ is a sequence of positive numbers such that $n^{-\frac{1}{2}} z_{n} \rightarrow \infty, n^{-(m-4) / 2} z_{n}$ remains bounded away from 0 , and $n^{-(m-2) / 2} z_{n} \rightarrow 0$ as $n \rightarrow \infty$, and

$$
B_{m}(n)=B_{m}^{*}(n)+O\left(z_{n}^{3-m}\right)
$$

for

$$
\begin{aligned}
B_{m}^{*}(n)= & \sum_{p=0}^{m-4} n^{-p} \sum_{i=0}^{2 p}\left(-n^{-\frac{1}{2}} z_{n}\right)^{-2 p+i}\left(\tilde{\sigma}_{n}^{-1} z_{n} h_{n}^{-1}\right)^{2 p-1} \sum_{q-1}^{j}(-1)^{q+p} c_{q j n} \\
& \times \frac{\{2(q+p)\} !}{2^{q+p}(q+p) !}
\end{aligned}
$$


where, for notational purposes, we define

$$
\sum_{q=1}^{0}(-1)^{q+p} c_{q 0 n} \frac{\{2(q+p)\} !}{2^{q+p}(q+p) !}=(-1)^{p} \frac{(2 p) !}{2^{p} p !},
$$

then $B_{m}(n)=1+O\left(n^{-(m-2) / 2} z_{n}\right)$ as $n \rightarrow \infty$.

Proof. We first note that $O\left(z_{n}^{3-m}\right)=O\left(n^{-(m-2) / 2} z_{n}\right)$. Next

$$
B_{m}^{*}(n)=1+\sum_{p=1}^{m-4} \sum_{j=0}^{2 p}\left(-\bar{\sigma}_{n}^{-1}\right)^{2 p-i} n^{-p}\left(n^{-\frac{1}{2}} h_{n}\right)^{j-2 p} K_{p j n}(m)
$$

where the numbers

$$
K_{p j n}(m)=\sum_{q=1}^{j}(-1)^{q+p} c_{q j n} \frac{\{2(q+p)\} !}{2^{q+p}(q+p) !}
$$

are bounded in absolute value by $K_{0}(m)$, a number depending on $m$ but not on $n$ (since the $c_{q j n}$ 's are uniformly bounded in $n$ ), $p$, or $j$. Therefore

$$
\left|B_{m}^{*}(n)-1\right| \leqq K_{0}(m) \sum_{p=1}^{m-4} \sum_{j=0}^{2 p} \bar{\sigma}_{n}^{j-2 p}\left(n^{-\frac{1}{2}} h_{n}\right)^{j} h_{n}^{-2 p} .
$$

In view of $(1.5 \mathrm{c})$ and $(1.5 \mathrm{~d})$, we can replace $K_{0}(m)$ by a constant $K_{1}(m)$ which differs only slightly and write, for sufficiently large $n$,

$$
\left|B_{m}^{*}(n)-1\right| \leqq K_{1}(m) \sum_{p=1}^{m-4} \sum_{j=0}^{2 p}\left(n^{-\frac{1}{2}} z_{n}\right)^{j} z_{n}^{-2 p} .
$$

Defining

$$
\theta_{n}(j, p)=\frac{\left(n^{-\frac{1}{2}} z_{n}\right)^{j} z_{n}^{-2 p}}{n^{-(m-2) / 2} z_{n}}
$$

we have only to show that $\theta_{n}(j, p)$ remains bounded under the assumption that $n^{-(m-4) / 2} z_{n}$ is bounded away from 0 as $n \rightarrow \infty$. But $m-5 \geqq m-3-2 p$ for $p \geqq 1$, and $j \leqq 2 p$, so that

$$
\begin{aligned}
\theta_{n}(j, p) & =\frac{\left(n^{\frac{1}{2}}\right)^{m-2 p-3}}{\left(n^{-\frac{1}{2}} z_{n}\right)^{2 p+1-j}} \leqq \frac{\left(n^{\frac{1}{2}}\right)^{m-5}}{n^{-\frac{1}{2}} z_{n}} \\
& \leqq\left\{n^{-(m-4) / 2} z_{n}\right\}^{-1}
\end{aligned}
$$

which remains bounded as $n \rightarrow \infty$ under the assumptions.

\section{The main theorem}

We have the following theorem on the probabilities of very large deviations of sums of iid random variables: 
(2.1) THEOREM. If $\left\{X_{n}: 1 \leqq n<\infty\right\}$ is a sequence of iid absolutely continuous random variables having $E\left(X_{1}\right)=0, \operatorname{Var}\left(X_{1}\right)=1, F(x)=P\left(X_{1} \leqq x\right), f(x)=$ $F^{\prime}(x), \phi(t)=E\left(\exp t X_{1}\right)$, and $Q(t)=\phi^{\prime}(t) / \phi(t)$ and satisfying the properties:

$$
\phi(t)<\infty \text { for all } t>0 ;
$$

(2.1b) for all sufficiently large real numbers $s$, the functions

$$
f_{s}(u)=\frac{e^{s u}}{\phi(s)} f(u)
$$

have uniformly bounded total variations $v_{s}, 0<v_{*} \leqq v_{s} \leqq v^{*}<\infty$;

$$
\lim _{t \rightarrow \infty} Q(t)=\infty ;
$$

(1.5b) there exist numbers $\sigma_{1}^{2}$ and $\sigma_{2}^{2}$ such that

$$
0<\sigma_{1}^{2} \leqq Q^{\prime}(t) \leqq \sigma_{2}^{2}<\infty
$$

for all sufficiently large $t$;

and

(1.12a) for an even positive integer $m \geqq 4$, there exist numbers $\gamma_{1}$ and $\gamma_{2}$ (depending on $m$ ) such that

$$
0<\gamma_{1} \leqq G^{(m)}(t) \leqq \gamma_{2}<\infty
$$

for all sufficiently large $t$, where

$$
G^{(m)}(t)=\sum_{j=0}^{m}\left(\begin{array}{c}
m \\
j
\end{array}\right)(-1)^{j} Q^{i}(t) \frac{\phi^{(m-i)}(t)}{\phi(t)},
$$

and $\left\{z_{n}: 1 \leqq n<\infty\right\}$ is a sequence of positive numbers such that $n^{-\frac{1}{2}} z_{n} \rightarrow \infty$, $n^{-(m-4) / 2} z_{n}$ remains bounded away from 0 , and $n^{-(m-2) / 2} z_{n} \rightarrow O$ as $n \rightarrow \infty$, then

$$
\begin{aligned}
P\left(n^{-\frac{1}{2}} S_{n} \geqq z_{n}\right)= & (2 \pi)^{-\frac{1}{2}} z_{n}^{-1} \\
& \times \exp \left\{-n^{-\frac{1}{2}} z_{n} Q^{-1}\left(n^{-\frac{1}{2}} z_{n}\right)+n \log \phi\left(Q^{-1}\left(n^{-\frac{1}{2}} z_{n}\right)\right)+b_{n}\right\} \\
& \times\left\{1+O\left(n^{-(m-2) / 2} z_{n}\right)\right\}
\end{aligned}
$$

where $S_{n}=\sum_{k-1}^{n} X_{k}$, and

$$
b_{n}=-\log \left\{n^{\frac{1}{2}} z_{n}^{-1} Q^{-1}\left(n^{-\frac{1}{2}} z_{n}\right) \sqrt{Q^{\prime}\left(Q^{-1}\left(n^{-\frac{1}{2}} z_{n}\right)\right)}\right\}
$$

is such that

$$
-\infty<\log \left(\sigma_{2}^{-1} \sigma_{1}^{2}\right) \leqq \varlimsup_{n \rightarrow \infty} b_{n} \leqq \log \left(\sigma_{1}^{-1} \sigma_{2}^{2}\right)<\infty .
$$

PRoof. In Lemma (1.2), we take $h=h_{n}$ and define 


$$
I_{n}=h_{n} \int_{0}^{\infty} e^{-h_{n} y}\left\{\bar{G}_{n}(y)-\bar{G}_{n}(0)\right\} d y .
$$

We first obtain a Cramér asymptotic expansion of the form (1.7h), where (1.13a) holds, of $\bar{G}_{n}(y)=P\left(\sum_{k=1}^{n} \bar{Y}_{n k} \leqq y\right)=P\left(\sum_{k=1}^{n} X_{n k} \leqq y \bar{\sigma}_{n}^{-1}\right)$ where $X_{n k}=$ $\bar{\sigma}_{n}^{-1} \bar{Y}_{n k}$. We proceed to verify the conditions of (1.7). Conditions (1.7a), (1.7b), and (1.7c) hold because of Lemma (1.5) and conditions (1.5a) and (1.5b) above. Condition (1.7d) is satisfied because of Lemma (1.9) and condition (1.12a) above and (1.5d). Conditions (1.7e) and (1.7f) follow from (2.1b) and the fact that $\bar{Y}_{n k}$ has density

$$
\bar{g}_{n k}(y)=n^{\frac{1}{2}} \frac{\exp \left\{n^{-\frac{1}{2}} h_{n}\left(n^{\frac{1}{2}} y+n^{-\frac{1}{2}} z_{n}\right)\right\}}{\phi\left(n^{-\frac{1}{2}} h_{n}\right)} f\left(n^{\frac{1}{2}} y+n^{-\frac{1}{2}} z_{n}\right)
$$

so that $v_{n k}=n^{\frac{1}{2}} v_{n}^{*}$, where $v_{n}^{*}$ is the total variation of the function $f_{s}(u)=$ $\left\{e^{s u} / \phi(s)\right\} f(u)$. Here $s=n^{-\frac{1}{2}} h_{n} \rightarrow \infty$ as $n \rightarrow \infty$ according to (1.5c). Finally, condition $(1.7 \mathrm{~g})$ follows from Lemma (1.12) and the fact that $v_{n k}$ does not depend on $k$ so that, for each $n,\{k: 1 \leqq k \leqq n\}$ is either $\Omega_{n}$ or $\Omega_{n}^{c}$. Therefore, setting $y=x \bar{\sigma}_{n}$, we have the asymptotic expansion

$$
\begin{aligned}
\bar{G}_{n}(y) & =\Phi\left(y \bar{\sigma}_{n}^{-1}\right)+\sum_{j=1}^{m-3} n^{-j / 2} P_{j n}(-\Phi)+R_{m n}\left(y \bar{\sigma}_{n}^{-1}\right) \\
& =H_{m n}\left(y \bar{\sigma}_{n}^{-1}\right)+R_{m n}\left(y \bar{\sigma}_{n}^{-1}\right)
\end{aligned}
$$

where, in expression $(1.13 \mathrm{c})$ for $P_{j n}(-\Phi), x$ is replaced by $y \bar{\sigma}_{n}^{-1}$ and by $(1.13 \mathrm{a})$, $\left|R_{m n}\left(y \bar{\sigma}_{n}^{-1}\right)\right| \leqq M n^{-(m-2) / 2}$. We can write

$$
I_{n}=I_{n}^{*}+O\left(n^{-(m-2) / 2}\right),
$$

where

$$
I_{n}^{*}=h_{n} \int_{0}^{\infty} e^{-h_{n} y}\left\{H_{m n}\left(y \bar{\sigma}_{n}^{-1}\right)-H_{m n}(0)\right\} d y .
$$

Using (1.13d), we can find the "characteristic function" of $H_{m n}\left(y \bar{\sigma}_{n}^{-1}\right)$, which is

$$
\gamma_{m n}\left(t \bar{\sigma}_{n}\right)=\sum_{j=0}^{m-3} n^{-j / 2} P_{j n}\left(i t \bar{\sigma}_{n}\right) \exp \left(-\frac{1}{2} t^{2} \bar{\sigma}_{n}^{2}\right)
$$

We define

$$
\begin{aligned}
f_{n}(y) & =e^{-h_{n} y} & & \text { for } y \geqq 0 \\
& =0 & & \text { for } y<0 .
\end{aligned}
$$

Then $g_{n}(t)=\int_{-\infty}^{\infty} e^{i t y} f_{n}(y) d y=\left(h_{n}-i t\right)^{-1}$ so by integration by parts and Parseval's formula 
[10]

$$
\begin{aligned}
(2 \pi)^{\frac{1}{2}} h_{n} I_{n}^{*} & =(2 \pi)^{-\frac{1}{2}} h_{n} \int_{-\infty}^{\infty} \bar{g}_{n}(t) \gamma_{m n}\left(t \bar{\sigma}_{n}\right) d t \\
& =(2 \pi)^{-\frac{1}{2}} \int_{-\infty}^{\infty} \frac{h_{n}}{h_{n}+i t}\left\{\sum_{j=0}^{m-3} n^{-j / 2} P_{j n}\left(i t \bar{\sigma}_{n}\right) \exp \left(-\frac{1}{2} t^{2} \bar{\sigma}_{n}^{2}\right)\right\} d t \\
& =\bar{\sigma}_{n}^{-1} \int_{-\infty}^{\infty}\left(1+\frac{i s}{h_{n} \bar{\sigma}_{n}}\right)^{-1}\left\{\sum_{j=0}^{m-3} n^{-i / 2} P_{j n}(i s)\right\} d \Phi(s),
\end{aligned}
$$

applying a change of variables $s=t \bar{\sigma}_{n}$. Now

$$
\left(1+\frac{i s}{h_{n} \bar{\sigma}_{n}}\right)^{-1}=\sum_{r=0}^{m-4}\left(-i s / h_{n} \bar{\sigma}_{n}\right)^{r}+\left(s / h_{n} \bar{\sigma}_{n}\right)^{m-3} w_{n}(s)
$$

where $\left|w_{n}(s)\right|$ is bounded in $n$ and $s$. Therefore

$$
\begin{aligned}
(2 \pi)^{\frac{1}{2}} h_{n} I_{n}^{*}= & \bar{\sigma}_{n}^{-1} \sum_{r=0}^{m-4} \sum_{j=0}^{m-3} \int_{-\infty}^{\infty}\left(-i s / h_{n} \bar{\sigma}_{n}\right)^{r} n^{-j / 2} P_{j n}(i s) d \Phi(s) \\
& +\bar{\sigma}_{n}^{-1} \sum_{j=0}^{m-3} \int_{-\infty}^{\infty}\left(s / h_{n} \bar{\sigma}_{n}\right)^{m-3} w_{n}(s) n^{-i / 2} P_{j n}(i s) d \Phi(s) \\
= & \bar{\sigma}_{n}^{-1} \sum_{j=0}^{m-3} n^{-j / 2} \sum_{r=0}^{m-4}\left(-h_{n} \bar{\sigma}_{n}\right)^{-r} \int_{-\infty}^{\infty}(i s)^{r} P_{j n}(i s) d \Phi(s) \\
& +O\left(h_{n}^{3-m}\right)
\end{aligned}
$$

by (1.13d) and the fact that $\Phi$ has finite moments of all orders. We now look at

$$
\mu_{r, j}=\int_{-x}^{\infty}(i s)^{r} P_{j n}(i s) d \Phi(s)
$$

From (1.13d), we have that

$$
(\text { is })^{r} P_{j n}(\text { is })=\sum_{q=1}^{j} c_{q j n}(i s)^{2 q+j+r} .
$$

Since odd moments of the standard normal distribution vanish, namely $\int_{-\infty}^{\infty} s^{2 p+1} d \Phi(s)=0$, it follows that $\mu_{r, j}=0$ whenever $j+r$ is odd. Recalling that $m$ is even, we can change variables as follows:

$$
\begin{aligned}
(2 \pi)^{\frac{1}{2}} h_{n} I_{n}^{*} & =\bar{\sigma}_{n}^{-1} \sum_{j=0}^{m-3} n^{-j / 2} \sum_{r+j=2 p} n^{-(2 p-r) / 2}\left(-h_{n} \bar{\sigma}_{n}\right)^{-r} \mu_{r, j}+O\left(h_{n}^{3-m}\right) \\
& =\bar{\sigma}_{n}^{-1} \sum_{p=0}^{m-4} \sum_{\substack{j=0 \\
(r+j=2 p)}}^{2 p} n^{-(2 p-r) / 2}\left(-h_{n} \bar{\sigma}_{n}\right)^{-r} \mu_{r, j}+O\left(h_{n}^{3-m}\right)
\end{aligned}
$$

where $r+j=2 p$ (since the terms vanish otherwise), and summing over $0 \leqq r+j \leqq 2 m-7$ and $0 \leqq p \leqq m-4$. Multiplying through by $z_{n} h_{n}^{-1}$, we obtain in view of $(1.5 c)$ that 


$$
(2 \pi)^{\frac{1}{2}} z_{n} I_{n}^{*}=\bar{\sigma}_{n}^{-1} z_{n} h_{n}^{-1} \sum_{p=0}^{m-4} n^{-p} \sum_{j=0}^{2 p}\left(-n^{-\frac{1}{2}} z_{n}\right)^{-2 p+j}\left(\bar{\sigma}_{n}^{-1} z_{n} h_{n}^{-1}\right)^{2 p-j} \mu_{2 p-j, j}+O\left(z_{n}^{3-m}\right)
$$

from which it follows that

$$
\begin{aligned}
(2 \pi)^{\frac{1}{2}} z_{n} I_{n}= & \bar{\sigma}_{n}^{-1} z_{n} h_{n}^{-1} \sum_{p=0}^{m-4} n^{-p} \sum_{j=0}^{2 p}\left(-n^{-\frac{1}{2}} z_{n}\right)^{-2 p+j}\left(\bar{\sigma}_{n}^{-1} z_{n} h_{n}^{-1}\right)^{2 p-j} \mu_{2 p-j, j} \\
& +O\left(z_{n}^{3-m}\right)+O\left(n^{-(m-2) / 2} z_{n}\right) .
\end{aligned}
$$

From (1.13d) and a knowledge of the even moments of the standard normal distribution, we find that

$$
\begin{aligned}
\mu_{2 p-j, j} & =(-1)^{p} \frac{(2 p) !}{2^{p} p !} & & \text { for } j=0 \\
& =\sum_{q=1}^{L}(-1)^{q+p} c_{q j n} \frac{2(q+p) !}{2^{q+p}(q+p) !} & & \text { for } 1 \leqq j \leqq 2 p .
\end{aligned}
$$

It follows from this and Lemma (1.2) that

$$
P\left(n^{-\frac{1}{2}} S_{n} \geqq z_{n}\right)=(2 \pi)^{-\frac{1}{2}}\left(h_{n} \bar{\sigma}_{n}\right)^{-1} e^{-z_{n} h_{n}} \phi^{n}\left(n^{-\frac{1}{2}} h_{n}\right)\left\{B_{m}(n)+O\left(n^{-(m-2) / 2} z_{n}\right)\right\}
$$

where $B_{m}(n)$ is as in Lemma (1.14). The proof is completed by applying Lemmas (1.14) and (1.5) and expression (1.6).

Some comments on the conditions before we close this section: condition (2.1b) plays a role akin to that of Cramér's Condition (C) for asymptotic expansions of distribution functions. For example, see pages 81 and 84 of Cramér's (1970) monograph or page 1020 of Bahadur and Ranga Rao's (1960) article.

Condition (1.5a) guarantees that the random variable $X_{1}$ is not a.s. bounded. If it were, then $P\left(n^{-\frac{1}{2}} S_{n} \geqq z_{n}\right)$ would be identically zero for all sufficiently large $n$. If $P\left(X_{1} \leqq c\right)=1$, then $c \phi(t)=c \int_{-\infty}^{c} e^{t x} d F(x) \geqq$ $\int_{-x}^{c} x e^{t x} d F(x)=\phi^{\prime}(t)$, so that $Q(t)=\phi^{\prime}(t) / \phi(t) \leqq c$.

Conditions (1.12a) and (1.5b), where $Q^{\prime}(t)=G^{(2)}(t)$, are moment conditions which serve to keep the tail probabilities of the same exponential order as normal tail probabilities. While it may be possible to relax these slightly, it seems that they form the normal analogue to the conditions in Heyde (1968), Ibragimov and Linnik (1971), and Rohatgi (1973) on the tail behavior of the underlying distribution function.

In Section 4 below, we will show that condition (2.1a) is necessary.

Finally, we show quickly that all conditions of Theorem 2.1 are satisfied by the normal distribution and that the assertion of the theorem reduces to the displayed equation at the beginning of Section 0 . If $\phi(t)=\exp \left\{\frac{1}{2} t^{2}\right\}$, then $Q(t)=t$ and $Q^{\prime}(t)=1$ so that conditions (2.1a), (1.5a), and (1.5b) hold with 
$\sigma_{1}^{2}=\sigma_{2}^{2}=1$. Furthermore, a tedious computation shows that $G^{(m)}(t)$ is constant for all $t$ and equal to $E\left(X_{1}^{m}\right)$ so that (1.12a) holds with $\gamma_{1}=\gamma_{2}=$ $E\left(X_{1}^{m}\right)$. Finally, condition (2.1b) is satisfied, because

$$
f_{s}(u)=(2 \pi)^{-\frac{1}{2}} \exp \left\{-\frac{1}{2}(s+u)^{2}\right\}
$$

so that $v_{*}=v^{*}=\sqrt{2 / \pi}$. In the conclusion of Theorem 2.1 we have $b_{n}=0$ and the other terms simplify as desired.

\section{A version of the Erdös-Rényi law}

If $\psi(x)$ is a continuous function of a real variable which increases monotonically to $\infty$ as $x \rightarrow \infty$, we will be interested in the almost sure behavior of

$$
\Sigma_{\psi}(N, K)=\max _{0 \leq n \leq N-K}\{K \psi(K)\}^{-\frac{1}{2}}\left(S_{n+k}-S_{n}\right)
$$

for various forms of $K$ (depending on $N$ ) as $N \rightarrow \infty$, where $S_{n}$ is the $n$th partial sum of a sequence of random variables. Erdös and Rényi (1970) opened up the study of random variables of the form $\Sigma_{\psi}(N, K)$ by showing what happens in the case $\psi(x)=x$. Some other aspects of the problem are discussed in the expository article Book (1976).

Before proceeding to the theorem itself, we derive from Theorem (2.1) some estimates of tail probabilities needed for the proof.

(3.1) COROllary. In the situation of Theorem (2.1), if $\psi$ is such that $x^{-1} \psi(x) \rightarrow \infty, x^{4-m} \psi(x)$ is bounded away from 0 , and $x^{2-m} \psi(x) \rightarrow 0$ as $x \rightarrow \infty$, then for every $\lambda>0$,

$$
\begin{aligned}
\sigma_{2}^{-1} \sigma_{1}^{2} \exp \left\{-\left(\lambda^{2} / 2 \sigma_{1}^{2}\right) \psi(n)\left(1+\left|\theta_{n}\right|\right)\right\} & \leqq 2\left\{2 \pi \lambda^{2} \psi(n)\right\}^{\frac{1}{2}} P\left\{n^{-\frac{1}{2}} S_{n} \geqq \lambda \sqrt{\psi(n)}\right\} \\
& \leqq 3 \sigma_{1}^{-1} \sigma_{2}^{2} \exp \left\{-\left(\lambda^{2} / 2 \sigma_{2}^{2}\right) \psi(n)\left(1-\left|\theta_{n}\right|\right)\right\}
\end{aligned}
$$

where $\theta_{n} \rightarrow \infty$ as $n \rightarrow \infty$.

Proof. In the conclusion of Theorem (2.1), we define

$$
w_{n}=n^{\frac{1}{2}} z_{n} Q^{-1}\left(n^{-\frac{1}{2}} z_{n}\right)-n \log \phi\left(Q^{-1}\left(n^{-\frac{1}{2}} z_{n}\right)\right)
$$

and we note that

$$
\left(\frac{1}{2} z_{n}^{2}\right)^{-1} w_{n}=2\left\{\frac{u_{n} Q\left(u_{n}\right)-\log \phi\left(u_{n}\right)}{Q^{2}\left(u_{n}\right)}\right\}=\frac{2 L\left(u_{n}\right)}{Q^{2}\left(u_{n}\right)}
$$

where $u_{n}=Q^{-1}\left(n^{-\frac{1}{2}} z_{n}\right) \rightarrow \infty$ as $n \rightarrow \infty$, and $L(t)=t Q(t)-\log \phi(t)$. Because $L^{\prime}(t)=t Q^{\prime}(t) \rightarrow \infty$ as $t \rightarrow \infty$, it follows from the fact that $L(0)=0$ that $L(t) \rightarrow \infty$ 
as $t \rightarrow \infty$. An application of L'Hôpital's Rule then shows that the limiting behavior of $2 L\left(u_{n}\right) / Q^{2}\left(u_{n}\right)$ to be the same as that of $\left\{Q^{\prime}\left(u_{n}\right)\right\}^{-1}$, from which it follows that

$$
\sigma_{2}^{-2} \leqq \varlimsup_{n \rightarrow \infty}\left(\frac{1}{2} z_{n}^{2}\right)^{-1} w_{n} \leqq \sigma_{1}^{-2}
$$

Therefore, for $n$ sufficiently large,

$$
\frac{1}{2} z_{n}^{2}\left(\sigma_{2}^{-2}-\left|\theta_{n}^{*}\right|\right) \leqq w_{n} \leqq \frac{1}{2} z_{n}^{2}\left(\sigma_{1}^{-2}+\left|\theta_{n}^{*}\right|\right)
$$

where $\theta_{n}^{*} \rightarrow 0$ as $n \rightarrow \infty$. The desired string of inequalities now follows from the conclusion of Theorem (2.1), upon setting $z_{n}=\lambda \sqrt{\psi(n)}$.

We now have the following extension of the Erdös-Rényi law of large numbers, the proof of which closely parallels that of the main theorem (Theorem 2.2) of Book (1975).

(3.2) THEOREM. If $\left\{X_{n}: 1 \leqq n<\infty\right\}$ is a sequence of iid random variables satisfying the conditions of Theorem (2.1), and $\psi(x)$ is a function of a real variable having the properties

(3.2a) $\psi(x)$ increases monotonically and continuously to $\infty$ as $x \rightarrow \infty$;

(3.2b) $\psi(x) / \psi(x+1) \rightarrow 1$ as $x \rightarrow \infty$; and

(3.2c) $\quad x^{-1} \psi(x) \rightarrow \infty, x^{4-m} \psi(x)$ is bounded away from 0 and $x^{2-m} \psi(x) \rightarrow 0$ as $x \rightarrow 0$,

then, for every $\lambda>0$,

$$
\underset{N \rightarrow \infty}{\lim \sup } \Sigma_{\psi}\left(N,\left[\psi^{-1}\left(2 \lambda^{-2} \sigma_{2}^{2} \log N\right)\right]\right) \leqq \lambda \quad \text { a.s. }
$$

and

$$
\liminf _{N \rightarrow \infty} \Sigma_{\psi}\left(N,\left[\psi^{-1}\left(2 \lambda^{-2} \sigma_{1}^{2} \log N\right)\right]\right) \geqq \lambda \quad \text { a.s. }
$$

where $[y]$ is the greatest integer not exceeding $y$.

If $X_{1}$ has the standard normal distribution, then $\sigma_{1}^{2}=\sigma_{2}^{2}=1$, and condition (1.12a) holds for all values of $m$. It follows that

$$
\lim _{N \rightarrow \infty} \Sigma_{\psi}\left(N,\left[\psi^{-1}\left(2 \lambda^{-2} \log N\right)\right]\right)=\lambda \quad \text { a.s. }
$$

whenever $x^{-1} \psi(x) \rightarrow \infty$ as $x \rightarrow \infty$.

\section{The necessity of the existence of the mgf for all $t>0$}

For a sequence of iid random variables having partial sums $\left\{S_{n}: 1 \leqq n<\right.$ $\infty$ \}, Baum, Katz, and Read (1962) proved that, if there are numbers $\lambda>0$, 
$C>0$, and $0<\rho<1$ such that $P\left(n^{-\frac{1}{2}} S_{n} \geqq \lambda \sqrt{n}\right) \leqq C \rho^{n}$ for all sufficiently large $n$, then there must exist a number $B, 0<B<\infty$, such that $\phi(t)=$ $E\left(\exp t X_{1}\right)<\infty$ for $0 \leqq t<B$. Their result was improved and sharpened by Petrov and Shirokova (1973).

It follows from Corollary (3.1) above that, under our conditions, $P\left\{n^{-\frac{1}{2}} S_{n} \geqq \lambda \sqrt{\psi(n)}\right\} \leqq C \rho^{\psi(n)}$ for all sufficiently large $n$. In this section, we show that condition (2.1a) is necessary for this latter bound to hold and therefore for the main theorem. In particular, using Petrov and Shirokova's methods, we have:

(4.1) THEOREM. If $\left\{X_{k}: 1 \leqq k<\infty\right\}$ is a sequence of iid random variables with partial sums $S_{n}=\sum_{k=1}^{n} X_{k}, g(n)$ is a monotonically increasing function of $n$ such that $n^{-1} g(n) \rightarrow \infty$ as $n \rightarrow \infty$, and there exist numbers $C>0$ and $0<\rho<1$ with

$$
P\left\{n^{-\frac{1}{2}} S_{n} \geqq \sqrt{g(n)}\right\} \leqq C \rho^{g(n)}
$$

for all sufficiently large $n$, then the $\operatorname{mgf} \phi(t)=E\left(\exp t X_{1}\right)<\infty$ for all $t>0$.

Proof. We first choose $\lambda>0$ so that $P\left(X_{1} \geqq-\lambda\right)>\frac{1}{2}(1+\rho)$, and we set $\theta=\max (\lambda, 1)$. Then

$$
P\left\{S_{n} \geqq \theta \sqrt{n g(n)}\right\} \geqq P\left\{X_{1} \geqq 2 \theta \sqrt{n g(n)}\right\} P\left\{S_{n-1} \geqq-\theta \sqrt{n g(n)}\right\} .
$$

But

$$
\begin{aligned}
P\left\{S_{n-1} \geqq-\theta \sqrt{n g(n)}\right\} & \geqq P\left\{\bigcap_{k=1}^{n}\left[X_{k} \geqq-\theta \sqrt{n g(n)}(n-1)^{-1}\right]\right\} \\
& \geqq\left\{P\left(X_{1} \geqq-\theta\right)\right\}^{n-1} \geqq\left\{\frac{1}{2}(1+\rho)\right\}^{n-1}
\end{aligned}
$$

since $\sqrt{n g(n)}(n-1)^{-1} \geqq 1$ for large $n$. Therefore

$$
\begin{aligned}
P\left\{X_{1} \geqq 2 \theta \sqrt{n g(n)}\right\} & \leqq C \rho^{g(n)}\left\{\frac{1}{2}(1+\rho)\right\}^{1-n} \\
& \leqq C\left\{2 \rho(1+\rho)^{-1}\right\}^{g(n)}=C \beta^{g(n)}
\end{aligned}
$$

where $\beta=2 \rho(1+\rho)^{-1}<1$, since $\left\{\frac{1}{2}(1+\rho)\right\}^{g(n)-n+1}<1$. Setting $\alpha=(2 \theta \beta)^{-1}$, we have that

$$
P\left\{X_{1} \geqq 2 \theta \sqrt{n g(n)}\right\} \leqq C \exp \{-2 \theta \alpha \sqrt{n g(n)} \sqrt{g(n) / n}\} .
$$

Now fix an arbitrarily large $x>0$ and denote by $n_{x}$ the largest integer such that $2 \theta \sqrt{n_{x} g\left(n_{x}\right)} \leqq x$. Then set $N_{x}$ to be the smallest integer such that $x \leqq 2 \theta \sqrt{N_{x} g\left(N_{x}\right)}$. Then for large $x$, 


$$
\begin{aligned}
P\left(X_{1} \geqq x\right) & \leqq P\left\{X_{1} \geqq 2 \theta \sqrt{n_{x} g\left(n_{x}\right)}\right\} \\
& \leqq C \exp \left\{-2 \theta \alpha \sqrt{N_{x} g\left(N_{x}\right)} \sqrt{\frac{n_{x} g\left(n_{x}\right)}{N_{x} g\left(N_{x}\right)}} \sqrt{g\left(n_{x}\right) / n_{x}}\right\} \\
& \leqq C \exp \left\{-\alpha x \sqrt{g\left(n_{x}\right) / n_{x}}\right\}
\end{aligned}
$$

Now, via an integration by parts, this implies that

$$
\begin{aligned}
\phi(t) & =\int_{-\infty}^{\infty} e^{i x} d F(x)=-\left.P\left(X_{1} \geqq x\right) e^{i x}\right|_{-\infty} ^{\infty}+t \int_{-\infty}^{\infty} e^{i x} P\left(X_{1} \geqq x\right) d x \\
& \leqq C t \int_{-\infty}^{\infty} e^{i x} e^{-\alpha x \sqrt{g\left(n_{x}\right) / n_{x}}} d x<\infty
\end{aligned}
$$

for all $t>0$ since $t-\sqrt{g\left(n_{x}\right) / n_{x}}<0$ for sufficiently large $x$.

\section{REFERENCES}

R. R. Bahadur and R. Ranga Rao (1960), 'On deviations of the sample mean', Ann. Math. Statist. 31, 1015-1027.

L. E. Baum, M. Katz, and R. R. Read (1962), 'Exponential convergence rates for the law of large numbers', Trans. Amer. Math. Soc. 102, 187-199.

S. A. Book (1970), Large deviations for order statistics and weighted sums (Ph.D. Dissertation, University of Oregon).

S. A. Book (1972), 'Large deviation probabilities for weighted sums', Ann. Math. Statist, 43, 1221-1234.

S. A. Book (1975), 'A version of the Erdös-Rényi law of large numbers for independent random variables', Bull. Inst. Math. Acad. Sinica 3, 199-211.

S. A. Book (1976), 'Large deviation probabilities and the Erdös-Rényi law of large numbers', Canadian J. Statist. 4, 185-209.

H. Cramér (1938), 'Sur un nouveau théoremè-limite de la théorie des probabilités, Actualités Scientifiques et Industrielles 736 (Hermann, Paris 5-23).

H. Cramér (1970), Random Variables and Probability Distributions, (3rd ed.), (Cambridge Univ. Press).

P. Erdös and A. Rényi (1970), 'On a new law of large numbers, J. Analyse Math. 23, 103-111.

W. Feller (1968), An Introduction to Probability Theory and Its Applications, 1 (3rd ed.), (Wiley, New York).

C. C. Heyde (1968), 'On large deviation probabilities in the case of attraction to a non-normal stable law', Sankhyä Ser. A. 30, 253-258.

I. A. Ibragimov and Yu. V. Linnik (1971), Independent and Stationary Sequences of Random Variables (Wolters-Noordhoff, Groningen).

V. V. Petrov (1954), 'Generalization of a limit theorem of Cramér', Uspekhi Mathem. Nauk. (N.S.) 9, 195-202. 
V. V. Petrov and I. V. Shirokova (1973), 'On exponential rates of convergence in the law of large numbers', Vestnik Leningrad. Univ. 2, 155-157.

V. K. Rohatgi (1973), 'On large deviation probabilities for sums of random variables which are attracted to the normal law', Comm. Statist. 2, 525-533.

Department of Mathematics,

California State College,

Dominguez Hills, California 90747. 